\title{
Imidazotriazinone Compounds
}

\section{Patent Highlight}

\section{David P. Rotella*}

Department of Chemistry and Biochemistry, Montclair State University, 1 Normal Avenue, Montclair, New Jersey 07043 , United States

Title:

Patent Application Number:

Priority Application:

Inventors:

Assignee Company:

Disease Area:

Summary:

Primary Markush:

Definitions:

Notable Substructures:

Biological Assay:

Biological Data:

Additional Information
Imidazotriazinone Compounds

WO 2012/040230

61/384694

Ripka, A.; Shapiro, G.; McRiner, A.

EnVivo Pharmaceuticals

Neurodegenerative
Biological Target:

Publication Date:

Priority Date:

Disease
This application claims a series of imidazotriazinone PDE9 inhibitors. PDE9 is a cGMP-selective phosphodiesterase that is implicated as a potential target in a range of CNS diseases including Alzheimer's Disease and other cognitive disorders.<smiles>[R][X]N1CC([R])C(c2nc([R2])n3nc([R])c4c(=O)[nH]c2c43)C1</smiles>

$\mathrm{X}=$ bond, carbonyl, or $\mathrm{SO}_{2}, \mathrm{R}_{1}=\mathrm{H}$, alkyl, cycloalkyl, heterocycloalkyl including substituted derivatives, $\mathrm{R}_{2}=$ cycloalkyl, heterocycloalkyl, phenyl, heteroaryl including substituted derivatives, $\mathrm{R}_{3}=$ alkyl, cycloalkyl, heterocycloalkyl including substituted derivatives.

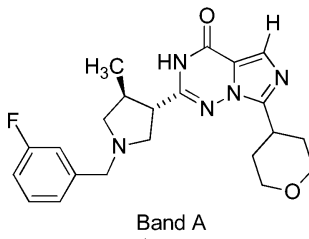

$>1000$-fold selectivity

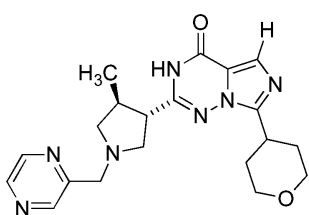

Band B

selectivity not determined

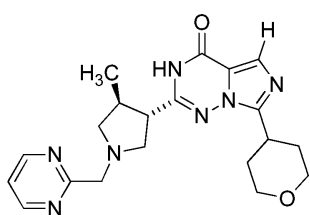

Band C

selectivity not determine
PDE assays were conducted using a Caliper LabChip instrument, and enzyme activity was measured spectrophotometrically to detect hydrolysis of a fluorescently labeled cyclic nucleotide. Selectivity against all other PDEs was measured. The patent reports fold selectivity versus PDE1A and PDE1B.

PDE inhibition data was provided by banding compounds: band A, IC50 $<1 \mu \mathrm{M}$; band B, 1-10 $\mu \mathrm{M}$; band C, $>10 \mu \mathrm{M}$.

PDE9 inhibitors are attracting attention for treatment of CNS disorders that can be influenced by elevation in cGMP levels. A PDE9 inhibitor, PF-04447943, is currently in clinical trials for cognitive disorders. The genus claimed in this application is reminiscent of the core structure of vardenafil, a PDE5 inhibitor related to sildenafil.

\section{AUTHOR INFORMATION}

\section{Corresponding Author}

*Tel: 973-655-7204. Fax: 973-655-7772. E-mail: davidprotella@ gmail.com.

Notes

The authors declare no competing financial interest. 